\title{
Loss of complex O-glycosylation impairs exocrine pancreatic function and induces MODY8-like diabetes in mice
}

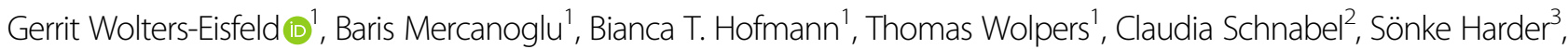
Pascal Steffen ${ }^{3}$, Kai Bachmann ${ }^{1}$, Babett Steglich', Jörg Schrader ${ }^{1}$, Nicola Gagliani ${ }^{1}$, Hartmut Schlüter ${ }^{3}$, Cenap Güngör ${ }^{1}$, Jakob R. Izbicki', Christoph Wagener ${ }^{4}$ and Maximilian Bockhorn ${ }^{1}$

\begin{abstract}
Cosmc is ubiquitously expressed and acts as a specific molecular chaperone assisting the folding and stability of core 1 synthase. Thus, it plays a crucial role in the biosynthesis of O-linked glycosylation of proteins. Here, we show that ablation of Cosmc in the exocrine pancreas of mice causes expression of truncated O-glycans (Tn antigen), resulting in exocrine pancreatic insufficiency with decreased activities of digestive enzymes and diabetes. To understand the molecular causes of the pleiotropic phenotype, we used Vicia villosa agglutinin to enrich Tn antigen-modified proteins from Cosmc-KO pancreatic lysates and performed a proteomic analysis. Interestingly, a variety of proteins were identified, of which bile salt-activated lipase (also denoted carboxyl-ester lipase, Cel) was the most abundant. In humans, frameshift mutations in CEL cause maturity-onset diabetes of the young type 8 (MODY8), a monogenic syndrome of diabetes and pancreatic exocrine dysfunction. Here, we provide data suggesting that differentially Oglycosylated Cel could negatively affect beta cell function. Taken together, our findings demonstrate the importance of correct O-glycan formation for normal exocrine and endocrine pancreatic function, implying that aberrant Oglycans might be relevant for pathogenic mechanisms of the pancreas.
\end{abstract}

\section{Introduction}

Among the multifarious spectrum of post-translational modifications, glycosylation is the most common and most diverse one. For some forms of glycosylation, there is substantial knowledge of their synthesis and biological functions. However, our knowledge of O-linked glycosylation is severely limited and represents an emerging field in research ${ }^{1}$. O-glycans are covalently $\alpha$-linked via an $N$ acetylgalactosamine (GalNAc) moiety to Ser and Thr residues $^{2,3}$ by the enzyme family of polypeptide $\mathrm{N}$ -

\footnotetext{
Correspondence: Gerrit Wolters-Eisfeld (ge.wolters@uke.de)

'Department of General, Visceral and Thoracic Surgery, University Medical Center Hamburg- Eppendorf (UKE), Hamburg, Germany

${ }^{2}$ Metabolic Laboratory and Newborn Screening, University Medical Center Hamburg-Eppendorf (UKE), Hamburg, Germany

Full list of author information is available at the end of the article.

These authors contributed equally: Gerrit Wolters-Eisfeld, Baris Mercanoglu,

Christoph Wagener, Maximilian Bockhorn
}

acetylgalactosaminyltransferases (ppGalNAcTs) to generate the O-glycan precursor, which is also called Tn antigen $(\mathrm{Tn})^{4}$. The most common modification of $\mathrm{Tn}$ is the formation of the core 1 ( $\mathrm{T}$ antigen) disaccharide catalyzed by $\mathrm{T}$-synthase ${ }^{5}$. The activity of T-synthase requires its chaperone $\mathrm{C} 1$ galt1c1 (hereafter Cosmc) ${ }^{6}$, which mediates proper protein folding in the endoplasmic reticulum (Fig. 1a) ${ }^{7}$.

The mammalian pancreas is a glandular organ with endocrine and exocrine functions. While endocrine cells are located in the islets of Langerhans and secrete insulin, glucagon, somatostatin and pancreatic polypeptide, the exocrine acinar cells produce inactive precursors of digestive enzymes that are stored in zymogen granules. After stimulation, zymogen granules migrate to the apical plasma membrane and release their contents into 

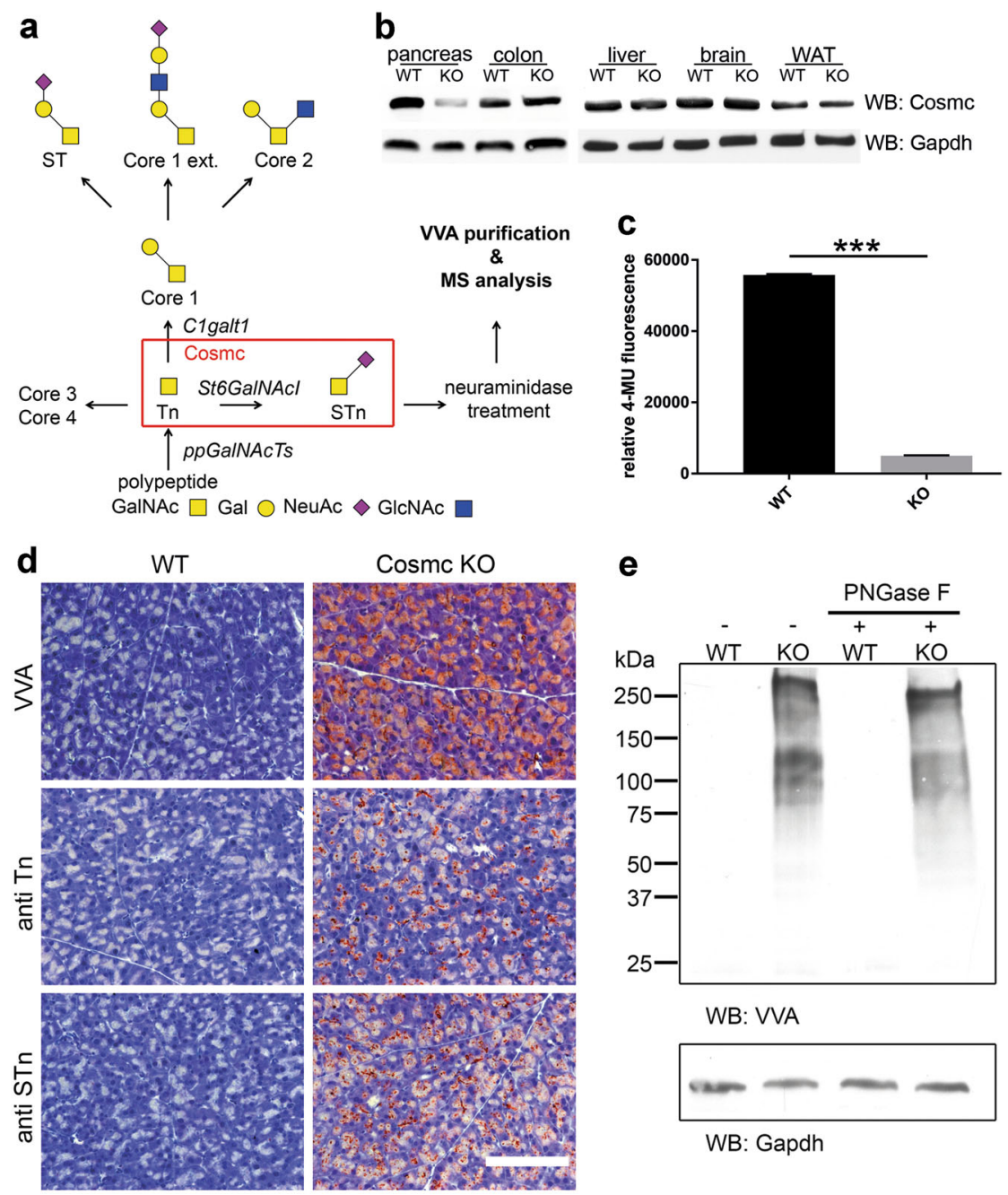

WB: Gapdh

Fig. 1 Deletion of Cosmc results in loss of core 1 glycan formation. a Scheme of O-glycosylation pinpointing the role of Cosmc. Depending on the biological context, a set of expressed ppGalNAcTs transfer GaINAc moieties to serine and threonine residues of the target proteins. Subsequent extension from Tn antigen to core 1 is performed by C1galt1 (T-synthase) being folded and stabilized by Cosmc. Lack of T-synthase activity results in Tn and STn expression. b Anti-Cosmc Western blot analysis of pancreas, colon, liver, brain and white adipose tissue (WAT) lysates derived from WT and pancreas-specific Cosmc-KO mice. Clearly reduced Cosmc protein is observed in KO pancreatic tissue. c The fluorescent T-synthase assay further validated reduced T-synthase activity in Cosmc-KO pancreatic tissue compared to WT $(n=4)$. $\mathbf{d}$ Representative histochemical staining using WA on FFPE WT and Cosmc-deficient mouse pancreata demonstrates specific binding to acinar cells in Cosmc KO. Further, antibodies directed against Tn and STn antigens specifically detected pancreatic acinar cells in Cosmc KO. The scale bar equals $50 \mu \mathrm{m}$. e Far-Western blot analysis of wholepancreatic tissue lysates of Cosmc-KO mice display Tn antigen expression on a variety of proteins, whereas no reactivity is observed in lysates of WT littermates. PNGase F treatment did not alter WA reactivity in Cosmc-KO lysates, emphasizing WA specificity towards O-linked GalNAc

pancreatic ducts that drain into the duodenum. Glycans participate in many key biological processes, including cell adhesion, molecular trafficking and clearance, receptor activation, signal transduction, and endocytosis ${ }^{8}$. On this basis, the role of $\mathrm{Tn}$ and its impact on pancreas physiology and disease was be investigated in this study. We generated a conditional floxed $\operatorname{Cosmc}$ mouse strain that was interbred with a pancreas-specific transcription factor 1 a (Ptf1a) Cre strain to obtain pancreatic Cosmc-KO offspring. Characterization of Cosmc-deficient mice revealed symptoms of exocrine pancreatic insufficiency with maldigestion, impaired zymogen granule release and decreased enzymatic elastase and lipase activity. Fasting serum glucose and glycated hemoglobin (HbA1c) were elevated, and fasting serum $\mathrm{C}$-peptide was reduced. Of note, cells of the endocrine pancreas were not affected by conditional Ptf1a-driven ${ }^{9}$ Cosmc ablation, suggesting molecular crosstalk between exocrine and endocrine cell populations ${ }^{10}$.

Cosmc deficiency and consecutive Tn antigen expression in the exocrine pancreas enables O-GalNAc glycoprotein identification using the plant lectin Vicia villosa 
agglutinin (VVA) ${ }^{11}$ for purification and subsequent massspectrometric proteome analysis ${ }^{12}$. It is assumed that differential O-glycosylation acts as the sum of all proteins involved, making Cosmc a powerful regulatory switch.

Carboxyl ester lipase, also known as bile salt-activated lipase, is highly expressed in pancreatic acinar cells. This digestive enzyme is activated by bile salts in the duodenum and participates in the hydrolysis and absorption of cholesterol and lipid-soluble vitamins ${ }^{13}$. The last of the 11 $C E L$ exons contains a variable number of tandem repeats (VNTR) region. Single-base deletions in the CEL VNTR cause an autosomal, dominantly inherited pancreatic disease characterized by diabetes and exocrine dysfunction, named maturity-onset diabetes of the young type 8 $(\mathrm{MODY} 8)^{14}$. CEL is heavily O-glycosylated at sites located in the VNTR at the protein C-terminus ${ }^{15}$. The VNTR is not required for functional properties of the enzyme, such as catalytic activity and activation by bile salts ${ }^{16}$. However, O-glycosylation has been suggested to be necessary for proper folding, secretion and stability ${ }^{17}$. Our data indicate that loss of core 1-derived O-glycans on Cel has effects analogous to nonsense point mutations causing MODY8like diabetes. This study demonstrates the impact of differential O-glycosylation on pancreas function and underscores the relevance of $\mathrm{O}$-glycosylation to pathophysiological mechanisms in vivo.

\section{Materials and methods}

\section{Generation of pancreatic acinar cell-specific Cosmc- knockout mice}

Based on the Cosmc cDNA sequence NM_021550 a targeting strategy was chosen, leading to the conditional deletion of Cosmc exon 2 by insertion of a lox site in intron 1 and a second lox site combined with an FRT-flanked neomycin selection cassette downstream of exon 2. This strategy resulted in the conditional deletion of the entire coding sequence. Male chimeras were bred, and heterozygous mice were generated. The conditional Cosmcknockout model was generated by genOway (Lyon, France). $\operatorname{Cosmc}^{\mathrm{fl} / \mathrm{fl}}$ females were crossed with Ptf1 ${ }^{\mathrm{cre} /+} ; \mathrm{Cosmc}^{-/ \mathrm{y}}$ males to generate homozygous Cosmc-KO and wild-type littermates. Homozygous Cosmc-KO male and female mice displayed the same phenotype. All mice were on a C57BL/6 $\mathrm{J}$ background. Genotyping was performed using the Kappa mouse genotyping hot start Kit (PeqLab, Erlangen, Germany). Genotyping primers were Cre-F 5'-ACCAGCC AGCTATCAACTCG-3, Cre-R 5-TTACATTGGTCCAG CCACC-3', Cosmc-F 5'- CACAGAACTCACTAT CCACTAGGCATGAATACAT-3'; Cosmc-R 5'-GCTCT CCCTAAATATACAACCGATTAAGAAAGTGT-3'. All animal experiments were performed in accordance with the National Institutes of Health Guide for the Care and Use of Laboratory Animals and were approved by the local animal ethics committee.

\section{Isolation and primary culture of islets and acinar cells}

Fresh pancreata were perfused with $3-5 \mathrm{ml}$ of collagenase (Sigma-Aldrich, St. Louis, MO, USA) solution in $0.5 \mathrm{mg} / \mathrm{ml} \mathrm{HBSS}$ on ice and incubated for $20 \mathrm{~min}$ at $5 \%$ $\mathrm{O}_{2}$ and $37^{\circ} \mathrm{C}^{18}$. Cells were carefully separated by pipetting up and down and washed with HBSS by centrifugation (500 $\mathrm{g}$ for $2 \mathrm{~min}$ ). Resuspended cells were poured through a $1000-\mu \mathrm{m}$ mesh, washed again and resuspended in $25 \%$ Ficoll solution. A Ficoll gradient was prepared by layering $2.4 \mathrm{ml}$ of each of 23, 20.5 and $11 \%$ Ficoll and centrifuging at $800 \mathrm{~g}$ for $15 \mathrm{~min}$. Islets of Langerhans were pelleted and washed in HBSS. Lysis was performed using RIPA buffer. Genomic DNA (gDNA) was purified using DNeasy Blood \& Tissue Kit (Qiagen, Hilden, Germany). Acinar cells were prepared and cultured at $1 \times 10^{6} / \mathrm{ml}$ in 24 -well plates and allowed to attach for $24 \mathrm{~h}^{19}$. The cells were treated with $10^{-8} \mathrm{M}$ cerulein (Sigma-Aldrich) for $2 \mathrm{~h}$. Cell culture supernatants were analyzed for levels of lipase and elastase.

\section{Isolation of pancreatic zymogen granules}

For isolation of pancreatic zymogen granules, pancreata were homogenized in homogenization buffer $(0.3 \mathrm{M}$ sucrose, $2 \mathrm{mM}$ MOPS, pH 6.8, Halt protease inhibitors, 1 $\mathrm{mM}$ EDTA) and centrifuged at $750 \mathrm{~g}$ for $10 \mathrm{~min}$ to remove cell debris and nuclei. Supernatants were filtered through $20-\mu \mathrm{m}$ nylon mesh and flow-through-centrifuged at $1750 \mathrm{~g}$ for $10 \mathrm{~min}$ to pellet zymogen granules ${ }^{20}$. Granules were lysed using RIPA buffer.

\section{Western blot, IHC/IF, VVA pulldown and real-time PCR}

These experiments were performed as we previously described $^{21-23}$. For Western blot, antibodies for GAPDH (sc-32233; Santa Cruz Biotechnology, Santa Cruz, CA, USA), Cosmc (sc-67480; Santa Cruz Biotechnology), CEL (ab79131; Abcam, Cambridge, UK; sc-34883; Santa Cruz Biotechnology), DMBT1 (MAB59151; R\&D Systems, Minneapolis, MN, USA), elastase (ab21593; Abcam) and trypsin (ab166898; Abcam) were used. Ten micrograms per milliliter of Vicia villosa lectin (VVA) reactive against O-GalNAc (Tn antigen) (B-1235; Vector Laboratories, Burlingame, CA, USA) complexed with $1 \mu \mathrm{g}$ streptavidinHRP (21126; Pierce, Thermo Fisher Scientific, Grand Island, NY, USA) was used. For immunohistochemistry, antibodies for Tn Antigen (MA180055; Thermo Fisher Scientific, Grand Island, NY, USA), STn Antigen (ab115957; Abcam), insulin (8138; CST, Beverly, MA, USA) and VVA-fluorescein (FL-1231; Vector Laboratories) were used at a dilution of $1: 100^{23}$. Ten micrograms per milliliter of Sambucus nigra lectin (SNA) (B- 1305-2; Vector Laboratories, Burlingame, CA, USA) was complexed with $1 \mu \mathrm{g}$ streptavidin-HRP. For real-time PCR, RNA was extracted with the RNeasy Plus Tissue Mini Kit (Qiagen). 


\section{Enzyme-linked immunosorbent assay (ELISA)}

All ELISAs were performed as proposed by the manufacturer (Cusabio, College Park, MD, USA). Mice were fasted for $4 \mathrm{~h}$ prior to blood collection. Mouse C-peptide was measured from undiluted plasma. Mouse glycated hemoglobin A1c (HbA1c) was measured from red blood cell lysates. Lipase and elastase were measured from total lysates and isolated zymogen granules ( $1 \mu \mathrm{g}$ total protein).

\section{Statistical analysis}

All data are expressed as the mean \pm SEM. Statistical analysis was performed with Prism 7.03 software (GraphPad Software, La Jolla, CA, USA). All data were tested for normality by applying the D'Agostino-Pearson omnibus test. If normality was confirmed and there were no significant differences in variance between groups, the two-tailed Student $t$ test was used to evaluate the differences.

\section{Quantification of islets}

IF staining for insulin was performed for $5 \mathrm{WT}$ and 5 Cosmc-deficient pancreata. Insulin-positive areas were measured with Keyence image analysis tool. For quantitation of Islets, ratios of islet area/total section area were log-transformed and analyzed by the Wilcoxon test. $P<0.05$ denoted a statistically significant difference.

\section{Biochemistry analyses}

Blood samples were centrifuged at 13,000 rpm for 10 min in a Biofuge 13 (Haereus, Hanau, Germany), and the analytes were determined from undiluted or 1:2-diluted serum (cholesterol, triglycerides, high-density lipoproteins). Samples were diluted with distilled water and were measured in a Dimension Vista 1500 (Siemens, Bad Nauheim, Germany). Analytes were measured by endpoint assays.

\section{Recombinant protein expression}

Recombinant 6xHIS tagged CEL and CEL-Tn (Clone ID: OHu22073C) was expressed in HEK293 and COSMCKO HEK293-SC (simple cells) ${ }^{3}$ and purified from Pro293 a Medium (Lonza, Basel, Switzerland) using Ni-NTA agarose (Qiagen).

\section{NT-3 insulin measurement and viability assay}

The cell line NT-3 was generated from a surgically resected lymph node of a 33-year-old male patient with a well-differentiated neuroendocrine tumor of the pancreas $^{24}$. Human insulin content was determined using the ADVIA Centaur Insulin Assay (REF 02230141) and ADVIA Centaur XP analyzer (Siemens Healthcare, Munich, Germany). This assay is standardized to the first human insulin international reference preparation by the World Health Organization (NIBSC code 66/304). Cell culture supernatants of NT-3 cells were analyzed after stimulation with $25 \mathrm{mM}$ Glucose for $60 \mathrm{~min}$ in the presence of $20 \mu \mathrm{M}$ recombinant CEL or CEL-Tn. Cell viability was assessed using the CellTiter-Glo luminescent cell viability assay (Promega, Madison, WI, USA) after 48 $\mathrm{h}$ of $20 \mu \mathrm{M}$ CEL or CEL-Tn treatment.

\section{Protein identification by mass spectrometry}

Tn modified proteins were identified by LC-MS/MS. Briefly, the enriched Tn-modified proteins were in-gel purified and excised from the polyacrylamide gel. MS/MS spectra obtained by collision-induced fragmentation after manual precursor selection were evaluated by the Mascot MS/MS search algorithm version 2.3 (Matrix Sciences, Boston, MA, USA) or SEQUEST HT.

\section{T-synthase and endogenous enzyme assays}

Ten micrograms of total protein from fresh tissue lysates was directly used to determine $T$-synthase activity using UDP-Gal (Sigma-Aldrich) as the donor and GalNAc- $\alpha-4$-methylumbelliferyl (GalNAc- $\alpha-4-M U)$ (Carbosynth, Compton, UK) as the acceptor ${ }^{25}$. OGlycosidase (New England Biolabs, Frankfurt am Main, Germany) was used for $\mathrm{T}$ antigen cleavage and release of fluorescent 4-MU. Fluorescence was measured (ex 355 $\mathrm{nm} / \mathrm{em} 460 \mathrm{~nm}$ ) in black OptiPlate-96 F plates (PerkinElmer, Waltham, MA, USA) in a FLUOstar Omega (BMG Labtech, Ortenberg, Germany). One microgram of total protein from fresh tissue lysates was directly used to determine lipase activity using 4-methylumbelliferyl butyrate (19362, Sigma-Aldrich) or pancreatic elastase using N-methoxysuccinyl-Ala-Ala-Pro-Val-7-amido-4methylcoumarin (M9771, Sigma-Aldrich) as substrate. Fluorescence was measured (ex $355 \mathrm{~nm} / \mathrm{em} 460 \mathrm{~nm}$ ) after $30 \mathrm{~min}$.

\section{Results}

To investigate the role of O-glycosylation in the function of the pancreas in vivo, we generated a conditional Cosmc flox mouse line that was interbred with a pancreas specific transcription factor 1a (Ptf1a)-Cre line to induce Tn antigen expression in pancreatic acinar cells (supplemental Fig. 1). A PCR-based genotyping protocol was established to identify wild-type (Cosmc WT) and activated hetero- $\left(\mathrm{Ptfla}^{+/ \mathrm{Cre}} ; \mathrm{Cosmc}^{+/ \mathrm{fl}}\right)$ and homozygous

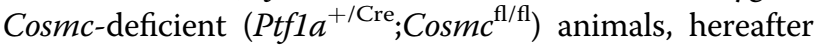
called Cosmc-KO (supplemental Fig. 2).

To verify the ablation of Cosmc on the mRNA and protein levels and the resulting enzymatic $\mathrm{T}$-synthase inactivity, we first probed whole pancreatic lysates with Cosmc-specific antibody. We observed a distinct decrease in Cosmc protein in Cosmc-KO-derived pancreas compared to WT, while in colon, liver, brain and white adipose tissue no difference was detectable (Fig. 1b). 
Table 1 Top 10 O-GalNac-modified proteins identified by VVA lectin precipitation coupled with LC-MS/MS proteome analysis. The complete table is available in the supplement

\begin{tabular}{llll}
\hline Accession & Protein & Gene & Score \\
\hline Q64285 & Bile salt-activated lipase & Cel & 516.25 \\
Q6P8U6 & Pancreatic triacylglycerol lipase & Pnlip & 471.75 \\
P10126 & Elongation factor 1-alpha 1 & Ef1a1 & 464.96 \\
P08113 & 78 kDa glucose-regulated protein & Grp78 & 444.59 \\
P20029 & Endoplasmin & Enpl & 360.26 \\
P00688 & Pancreatic alpha-amylase & Amy2 & 325.07 \\
Q9DBG6 & Dolichyl-diphosphooligosaccharide-- & Rpn2 & 296.01 \\
& protein glycosyltransferase subunit 2 & & \\
Q8VDJ3 & Vigilin & Hdllbp & 285.34 \\
Q9JKR6 & Hypoxia upregulated protein 1 & Hyou1 & 233.90 \\
O88986 & 2-Amino-3-ketobutyrate coenzyme A & Gcat & 224.19 \\
& ligase, mitochondrial & & \\
\hline
\end{tabular}

Corresponding results were obtained when Cosmc mRNA expression in the pancreas was measured by quantitative RT-PCR (supplemental Fig. 3). Compared to WT, Cosmc mRNA was reduced by greater than $80 \%$ in Cosmc-KO mice. Interestingly, we found that the T-synthase gene was overexpressed in the pancreas of Cosmc-KO mice, suggesting a possible regulative feedback mechanism (supplemental Fig. 3). Ultimately, we tested T-synthase activity in pancreatic lysates derived from WT and Cosmc-KO mice. The results clearly indicate dysfunctional $\mathrm{T}$ synthase in Cosmc-KO mice compared to WT (Fig. 1c).

In proof-of-concept experiments, we further subjected pancreata of WT and Cosmc-KO mice to histological staining using monoclonal antibodies and specific lectins directed against Tn and STn (Fig. 1d). Immunohistochemical staining using streptavidin-horseradish peroxidase complexed with biotinylated VVA on formalinfixed, paraffin-embedded WT and Cosmc-KO mouse pancreata demonstrated specific binding of VVA in exocrine glandular cells of Cosmc-KO mice. Further, antibodies directed against $\mathrm{Tn}$ and STn antigens specifically bound to Cosmc-KO pancreatic acinar cells, indicating that a relevant portion of expressed Tn antigen was sialylated.

Immunofluorescence using fluorescein-VVA confirmed specific binding to pancreatic acinar cells in Cosmc-KO tissue (supplemental Fig. 4). Western and far-Western blot analysis of whole-pancreatic tissue lysates of CosmcKO mice displayed Tn antigen expression on a variety of proteins, whereas no reactivity was observed in lysates from WT littermates (Fig. 1e). We additionally used biotinylated lectin HPA and anti-Tn antigen antibody for
Tn antigen detection on O-GalNAc-modified glycoproteins on Cosmc-KO pancreatic acinar cells (supplemental Fig. 5). In addition, WT and Cosmc-KO pancreatic tissue lysates were treated with $\mathrm{N}$-glycosidase $\mathrm{F}$ (PNGase F) to remove $\mathrm{N}$-glycans. PNGase $\mathrm{F}$ treatment did not alter VVA reactivity in Cosmc-KO lysates (Fig. 1e).

We next employed VVA-based enrichment of Tnmodified glycoproteins with subsequent LC-MS/MS analysis. Cel is one of the highly abundant, differentially $\mathrm{O}$-glycosylated proteins expressed in the murine exocrine pancreas (Table 1). Cel belongs to the type-B carboxylesterase/lipase family and possesses a C-terminal domain with VNTR, a PEST motif and a high proportion of O-glycan moieties (Fig. 2a) ${ }^{14}$. Western blot analysis of pancreatic lysates using a Cel antibody showed bands at approximately 65,75 and $120 \mathrm{kDa}$. VVA pulldown and subsequent detection with the same Cel antibody displayed a prominent band specifically in the Cosmc-KO sample at $65 \mathrm{kDa}$ (Fig. 2b). Further experimentally validated $\mathrm{Tn}$-modified proteins are shown in Fig. 3c and supplemental Figure 6.

To better understand the role of O-glycosylation on exocrine pancreatic function, we quantitated protein levels of pancreatic lipase and elastase (Ela2a) in total lysates and zymogen granules using ELISA. Pancreatic lipase had similar expression levels between WT and Cosmc-KO in total lysates but was elevated in Cosmc-KO zymogen granules (Fig. 2c). Elastase was significantly increased in both total lysates and zymogen granules of Cosmc KO (Fig. 2d). Analysis of relative lipase and elastase activities in zymogen granules normalized to actual lipase and elastase protein content revealed significantly reduced activities in Cosmc-deficient mice (Figs. 2e, f), indicating a direct impact of aberrant O-GalNAc glycosylation on enzymatic activity/activation. To evaluate the impact of core-1 derived O-glycans on physiological exocrine potential, primary pancreatic acinar cells derived from fasted WT and Cosmc-KO animals were prepared, cultured and cerulein stimulated. Cerulein is a gastroregulatory molecule with actions similar to cholecystokinin and thus induces the release of digestive enzymes from the pancreas. Subsequently, lipase and elastase activities from cell culture supernatants were measured using a specific 4-MU substrates (Figs. 2g, h). The stimulatory response to cerulein in WT acinar cells was apparent, whereas a significant reduction was obvious in Cosmc-KO cells.

To analyze the possible impact of aberrant Oglycosylation on ER function as well as zymogen granule formation and maturation, adult and fasting WT and Cosmc-KO pancreata $(n=3)$ were subjected to transmission electron microscopic analysis (Fig. 3a). No gross abnormalities were obvious, and the ER had a normal structure, indicating no ER stress induced by protein 

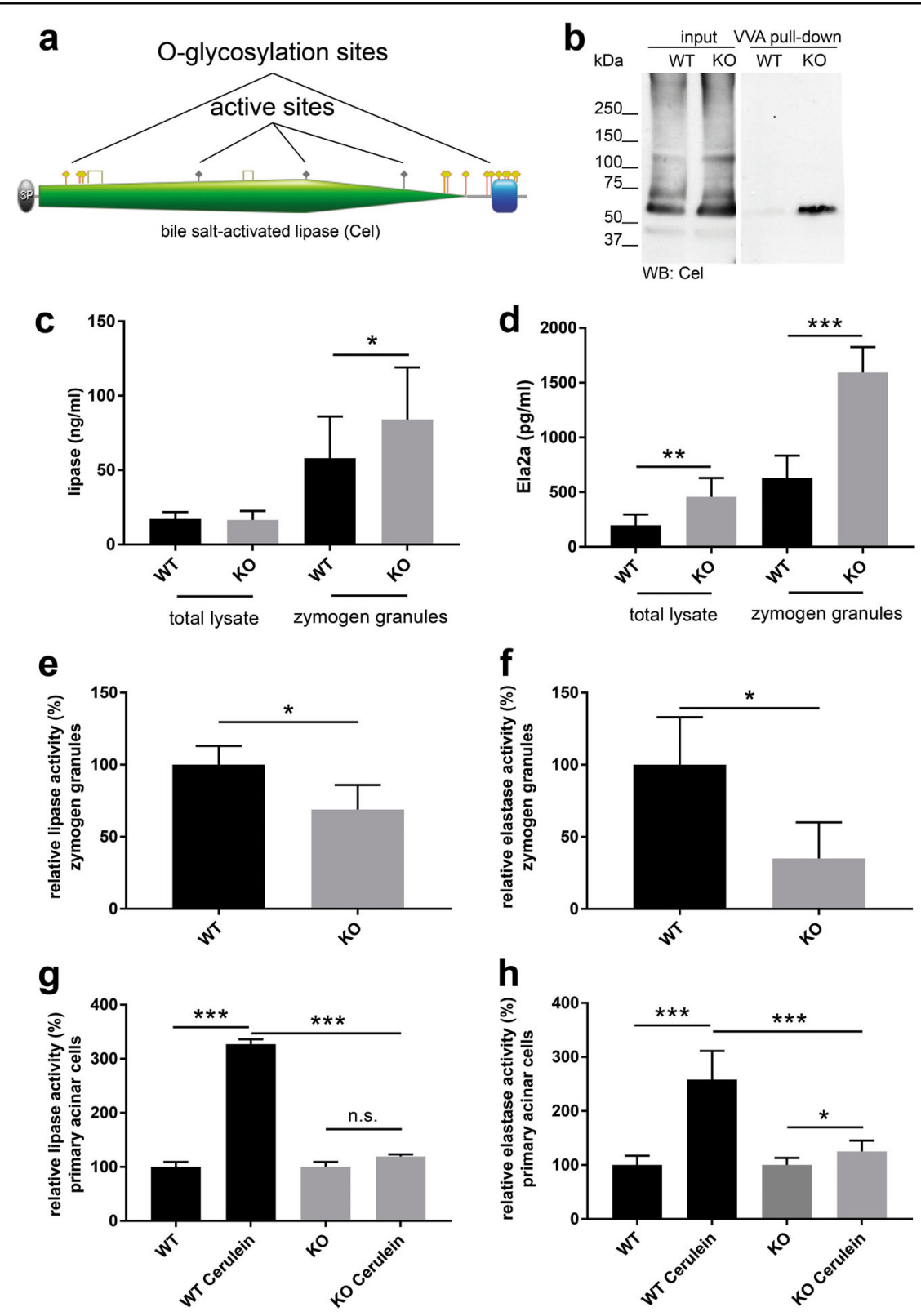

Fig. 2 Cosmc-dependent O-GalNAc glycosylation affects pancreatic lipase and elastase enzyme function as well as exocrine pancreas secretion. a Scheme of bile salt-activated lipase (Cel) protein domains (SP, signal peptide; carboxylesterase, type B, green; PEST domain, blue; OGalNAc glycosylation sites, yellow; active sites, gray). b Representative WB of WA pull-down from WT and Cosmc-KO pancreatic lysates and Celspecific antibody for detection. Note: Distinct Cel protein bands were robustly detected in pull-downs from KO tissue. c Absolute quantification of lipase in total lysates and zymogen granule lysates from WT and Cosmc-KO mice $(n=18)$. Total lysates display equal amounts of lipase $(P=0.748)$, whereas elevated levels are detected in Cosmc-KO zymogen granules $(P=0.023)$. $\mathbf{d}$ Absolute quantification of elastase (Ela2a) in total and zymogen granule lysates from WT and Cosmc-KO mice $(n=18)$. Lysates derived from Cosmc KO display significantly elevated elastase. (e $+\mathbf{f})$ Relative lipase and elastase activities in zymogen granule lysates from WT and Cosmc-KO mice $(n=4)$ using 4-MU substrates reveal decreased activities in KO. $(\mathbf{g}+\mathbf{h})$ Analysis of lipase and elastase activities in response to cerulein stimulation of WT and Cosmc-KO primary pancreatic acinar cells. Ceruleininduced lipase and elastase secretion is detectable in WT cells $(P=0.0001)$ and is attenuated in Cosmc-KO mice $(P=0.611$ and $P=0.04)$. Relative difference in stimulated lipase activity is $P=0.0001$

misfolding and/or ER-associated degradation ${ }^{26}$. However, the number of zymogen granules in Cosmc-deficient pancreata was considerably increased. Systematic counting of electron-dense zymogen granules confirmed a significant three-fold elevation in Cosmc-KO mice compared to WT (Fig. 3b). Among the identified Tn antigenmodified glycoproteins, we found the known heavily $\mathrm{N}$ - and O-glycosylated mucin-like protein DMBT1 

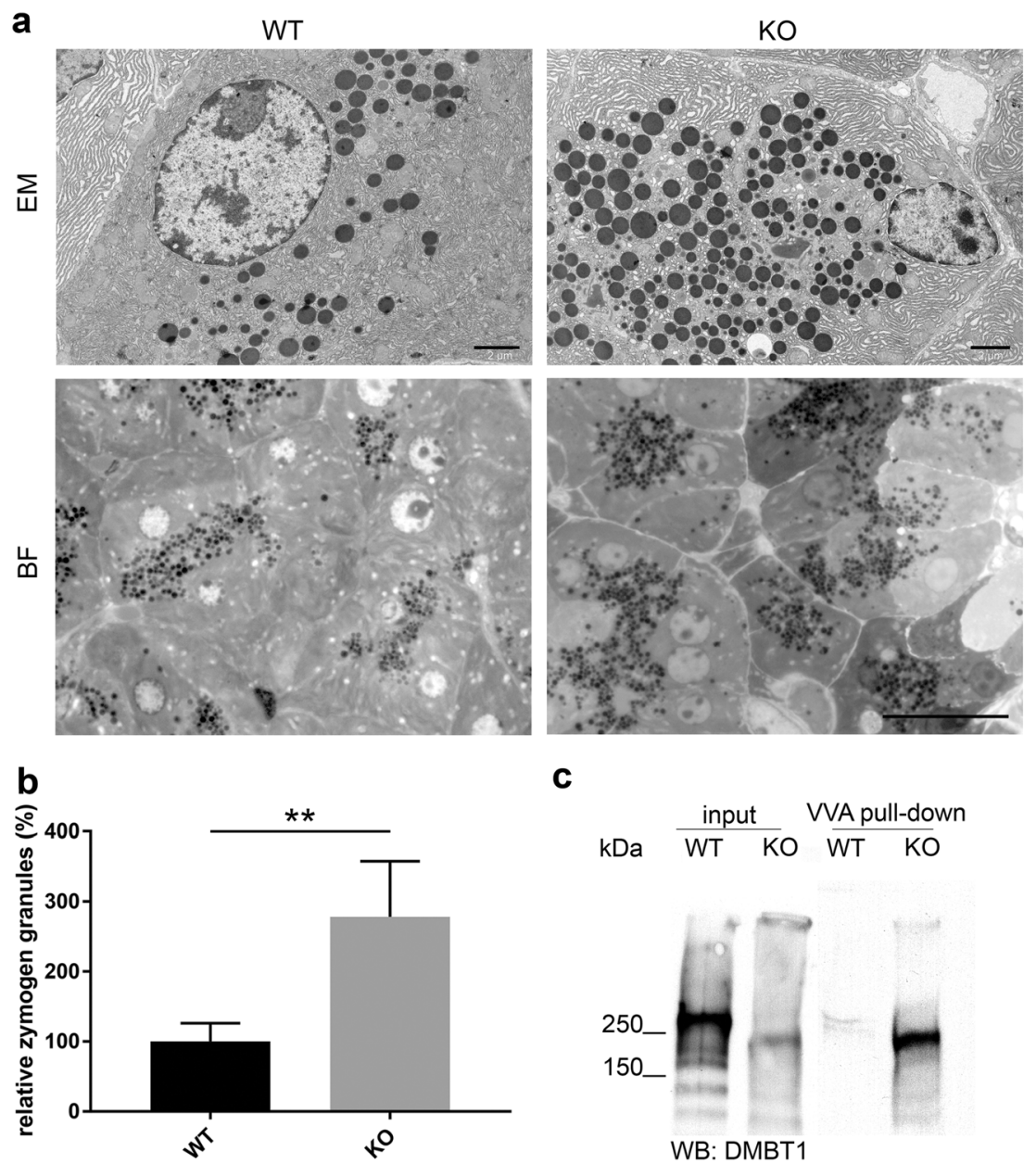

C
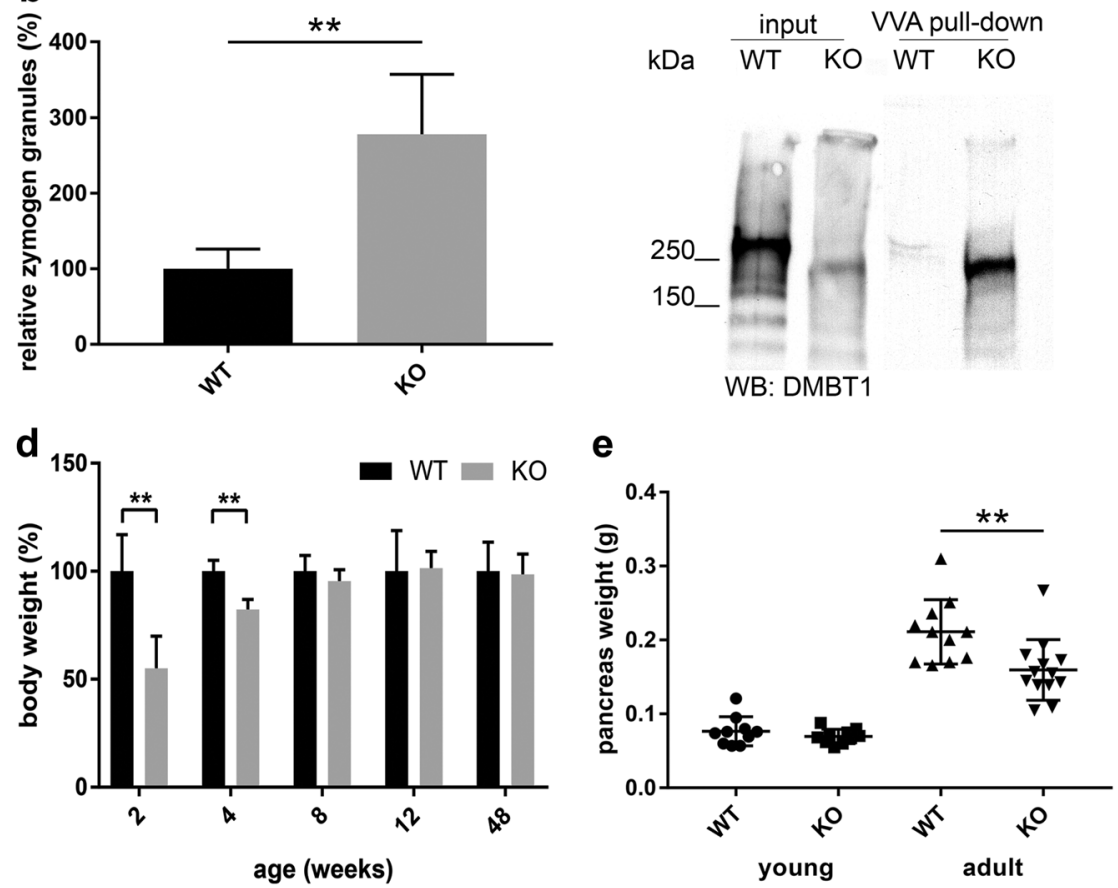

Fig. 3 Cosmc deficiency results in accumulation of zymogen granules and pancreas atrophy in aged mice. a Representative TEM and brightfield micrographs of pancreas sections from fasting WT and Cosmc-KO adult mice. Zymogen granules are depicted as electron dense vesicles. TEM and BF scale bars are 2 and $20 \mu \mathrm{m}$, respectively. $\mathbf{b}$ Quantification of zymogen granules in five randomly selected fields of pancreas sections from fasting WT and Cosmc-KO adult mice ( $n=5$ each). Elevated numbers of zymogen granules in Cosmc-KO pancreata $(P=0.0004)$. $\mathbf{c}$ WB of WA pulldown from WT and Cosmc-KO pancreatic lysates and DMBT1-specific antibody for detection. $\mathbf{d}$ Body weight analysis of WT and Cosmc-KO mice at ages 2-48 weeks ( $n=10$ each) displays reduced body weight gain at 2 and 4 weeks of age $(P=0.0021$ and $P=0.0002)$. e Assessment of pancreas organ weight of 20- $(n=10)$ and 54-week-old WT $(n=11)$ and Cosmc-KO $(n=13)$ mice. Aged mice display reduced organ weight $(P=0.0062)$

(deleted in malignant brain tumors 1). DMBT1 has been described as a structural zymogen granule protein involved in the regulation of digestive enzyme secretion. It was subjected to Western blot analysis, which revealed a notable difference in the apparent DMBT1 molecular weight between WT and Cosmc KO. The down-shift in 
Table 2 Laboratory values of WT and Cosmc-KO mouse blood samples ( $n=5$ each). Unpaired two-tailed Student's $t$-test was used to test the level of significance

\begin{tabular}{llllc}
\hline Characteristic & Wild-type (mean \pm SD) & Cosmc KO (mean \pm SD) & P value & $\mathbf{9 5 \% ~ C l ~}$ \\
\hline Total cholesterol $(\mathrm{mg} / \mathrm{dl})$ & $129.3 \pm 11.7$ & $103.2 \pm 7.1$ & 0.007 & $9.95-42.05$ \\
Triglycerides $(\mathrm{mg} / \mathrm{dl})$ & $110.0 \pm 21.6$ & $94.4 \pm 10.8$ & 0.211 & $-11.69-42.89$ \\
High-density lipoprotein $(\mathrm{mg} / \mathrm{dl})$ & $152.0 \pm 4.0$ & $96.4 \pm 15.4$ & 0.001 & $36.61-82.58$ \\
\hline
\end{tabular}

$\mathrm{KO}$ lysates clearly underscores the loss of complex Oglycosylation. For validation, VVA pull-down discriminated WT from Cosmc-KO DMBT1 glycoforms (Fig. 3c).

Exploration of the small and large intestine of nonfasting WT mice revealed semisolid stool in the distal small intestine and fully formed pellets of stool in the proximal colon. In contrast, Cosmc-KO mice displayed a brightened, semisolid stool in the distal colon, indicating insufficient digestion and malabsorption. A laboratory analysis of blood samples from WT and Cosmc-KO animals revealed differences in lipid profiles related to exocrine pancreatic function, with decreased total cholesterol, triglycerides and high-density lipoprotein (HDL) in Cosmc-KO animals (Table 2).

Furthermore, we monitored body weights of male WT and Cosmc-KO littermates at 2, 4, 8, 12, 24 and 48 weeks of age. A significant decrease in body weight gain in young ( 2 and 4-week-old) Cosmc-KO animals was observed. This phenotype was reversed at 8 weeks of age and was no longer observable at later time points (Fig. 3d). The Cosmc-KO pups did not exhibit signs of negligence, abnormal coat state or poor breast-feeding behavior. Pathological assessment of hematoxylin and eosin (HE)stained WT and Cosmc-KO pancreatic sections revealed that pancreatic organ development was unaltered, and no gross abnormalities were observed. However, 1-year-old Cosmc-KO mice displayed pancreatic atrophy (Fig. 3e and supplemental Figure 7).

Having identified Cel as being critically involved in MODY8 pathogenesis as a major Tn-modified pancreatic protein, we analyzed glucose metabolism in WT and Cosmc-KO mice. First, fasting serum glucose was measured in a large cohort of young and adult mice, with significantly elevated glucose observed in aged Cosmc-KO and not in young mice (Fig. 4a). This result was further validated by the measurement of glycated hemoglobin (HbA1c), which was significantly elevated in adult mice, indicating long-term hyperglycemia (Fig. 4b). To further pinpoint the molecular mechanism of the observed diabetes, we assayed insulin (Ins1 and Ins2) expression levels in total pancreas derived from WT and Cosmc-KO animals. To our surprise, we found that both insulin genes were significantly upregulated in Cosmc-KO animals
(Fig. 4c). As a key regulator of glucose metabolism, insulin is subjected to regulated proteolytic activation, resulting in the generation of insulin C-peptide. Thus, C-peptide level in the circulation reflects insulin activation. Additionally, ELISA-based quantification of C-peptide from sera of WT and Cosmc-KO mice revealed a significantly decrease in the latter (Fig. 4d).

Next, we analyzed the expression of insulin/proinsulin and Tn antigen by immunohistochemistry in pancreata from fasting young and adult WT and Cosmc-KO mice. Consistent with previous reports, Ptfla-Cre driven Cosmc-KO resulted in Tn antigen positivity restricted to pancreatic acinar cells in young animals (Fig. 5a). Insulinproducing beta cells were equally stained in WT and Cosmc-KO tissue in young mice. Interestingly, pronounced Tn antigen positivity was observed in islets of Langerhans in adult Cosmc-KO mice. To verify that the observed Tn positivity in the islets of Langerhans derived from exocrine proteins, we stained WT and $\mathrm{KO}$ tissue for Cel (Fig. 5b). Interestingly, KO tissue displayed marked Cel positivity compared to WT. This result was further substantiated by immunofluorescence co-staining of Tn and $\mathrm{Cel}$ in $\mathrm{KO}$ islets of Langerhans (Fig. 5b lower panel). Quantification of insulin-positive islet areas (islet area/ total section area) revealed a significant reduction in $\mathrm{KO}$ pancreata (Fig. 5c). Analysis of Tn antigen expression revealed positivity and a distinct binding pattern in lysates derived from Cosmc-KO pancreatic islets. Anti-trypsin antibody was used to evaluate cross-contamination of exocrine-derived proteins in the endocrine compartment (Fig. 5d). It was apparent that elevated levels of Cel were found in islets of Langerhans derived from Cosmc-KO animals. This result is consistent with the staining presented in Fig. 5b. We next extracted genomic DNA from freshly isolated pancreatic islets and found no Cosmc deletion in endocrine cells, in contrast to exocrine tissue, by PCR (supplemental Figure 8 ). This result clearly demonstrates that $\mathrm{Tn}$ antigen positivity observed in pancreatic islets was not due to Cre-mediated Cosmc excision. We further analyzed cell lysates from isolated pancreatic islets and were able to detect Cel by Western blot in tissues derived from WT and Cosmc-KO animals. $\mathrm{Cel}$, detected in lysates from Cosmc-KO endocrine pancreas, showed a characteristic staining pattern of 

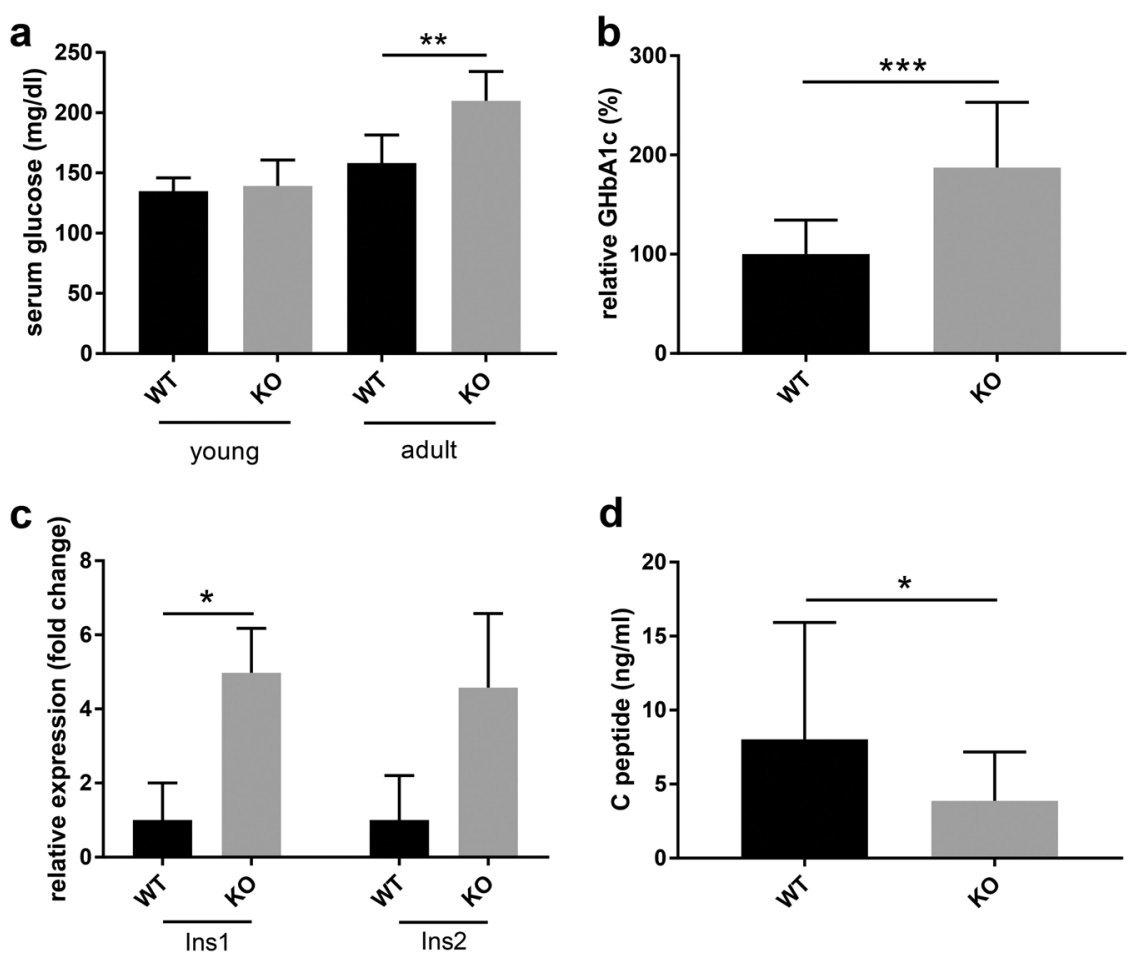

Fig. 4 Exocrine pancreatic Cosmc KO induces diabetes in adult mice. a Measurement of fasting serum blood glucose in young animals $(n=10$; $P=0.58)$ and adult mice $(n=15 ; P=0.0005)$. $\mathbf{b}$ Quantification of HbA1c confirms diabetes in adult Cosmc-KO mice $(n=15$ each; $P<0.0001)$. c Quantitative PCR analysis of Ins1 and Ins2 from total WT and Cosmc-KO pancreas mRNA $(n=3)$ indicates insulin overexpression by a factor of 5.0 $(P=0.025)$ and a factor of $4.58(P=0.053)$, respectively, in Cosmc KO. $\mathbf{d}$ Absolute C-peptide quantification in sera from WT $(n=18)$ and Cosmc-KO $(n=19)$ adult mice shows reduced C-peptide expression in Cosmc-KO mice $(P=0.043)$

additional high-molecular-weight bands compared to WT (Fig. 5e). In addition, we specifically detected Cel after VVA precipitation from lysates derived from Cosmc-KO pancreatic islets by Western blot (Fig. 5f). Lastly, we assayed the impact of CEL O-glycotype on cell viability and insulin secretion in vitro. We used human CEL recombinantly expressed in HEK293 WT and HEK293 simple cells $(\mathrm{SC})^{3}$ to treat NT-3 cells ${ }^{24}$, an insulin-producing human neuroendocrine tumor cell line, in the presence of glucose. Comparative analysis of the impact of CEL-Tn and CEL on NT-3 cell viability displayed a trend after $48 \mathrm{~h}$, wherein CEL-Tn reduced cell viability (Fig. 5g). Further, measurement of glucosestimulated insulin secretion clearly indicated that CELTn derived from HEK293 SC negatively regulated secretion compared to CEL with normal glycotype (Fig. 5h).

\section{Discussion}

Cosmc is the essential chaperone for correct protein Oglycosylation, which in turn is essential for correct cellular function. Depletion of Cosmc results in embryonic lethality, highlighting its role in developmental processes ${ }^{6}$. Cosmc is ubiquitously expressed, but the biological functions of $\mathrm{O}$ - glycosylation have not been elucidated globally. The main reason for this is biological heterogeneity of cell types and tissues, leading to inconsistent phenotypes when aberrant $\mathrm{O}$-glycosylation is induced by genetically switching off Cosmc or T-synthase. Recent examples are altered integrity of the glomerular filtration barrier, resulting in spontaneous proteinuria and rapidly progressing glomerulosclerosis ${ }^{27}$ and a compromised mucus layer, spontaneous microbe-dependent inflammation, and colitis $^{28}$. Correct O-glycosylation in pancreatic acinar cells is required for normal zymogen granule formation and regulated protein secretion in vitro ${ }^{29}$. When O-linked glycosylation is perturbed, the secretory pathway is inhibited, accompanied by morphological alterations due to the aggregation of zymogen granules and association of aggregates with the trans-Golgi network membrane. Interestingly, DMBT1 has been identified as a structural zymogen granule protein involved in the regulation of the secretory pathway ${ }^{30,31}$. Prerequisite to secretion is functional protein folding in ER, mediated by multiple chaperoning systems. The $78-\mathrm{kDa}$ glucose regulated protein (GRP78) is a general chaperone recognizing unfolded proteins by its hydrophobic residues ${ }^{32}$. GRP78 is O-glycosylated in many cancer cell lines, but GRP78 O-glycosylation is not linked to the unfolded 


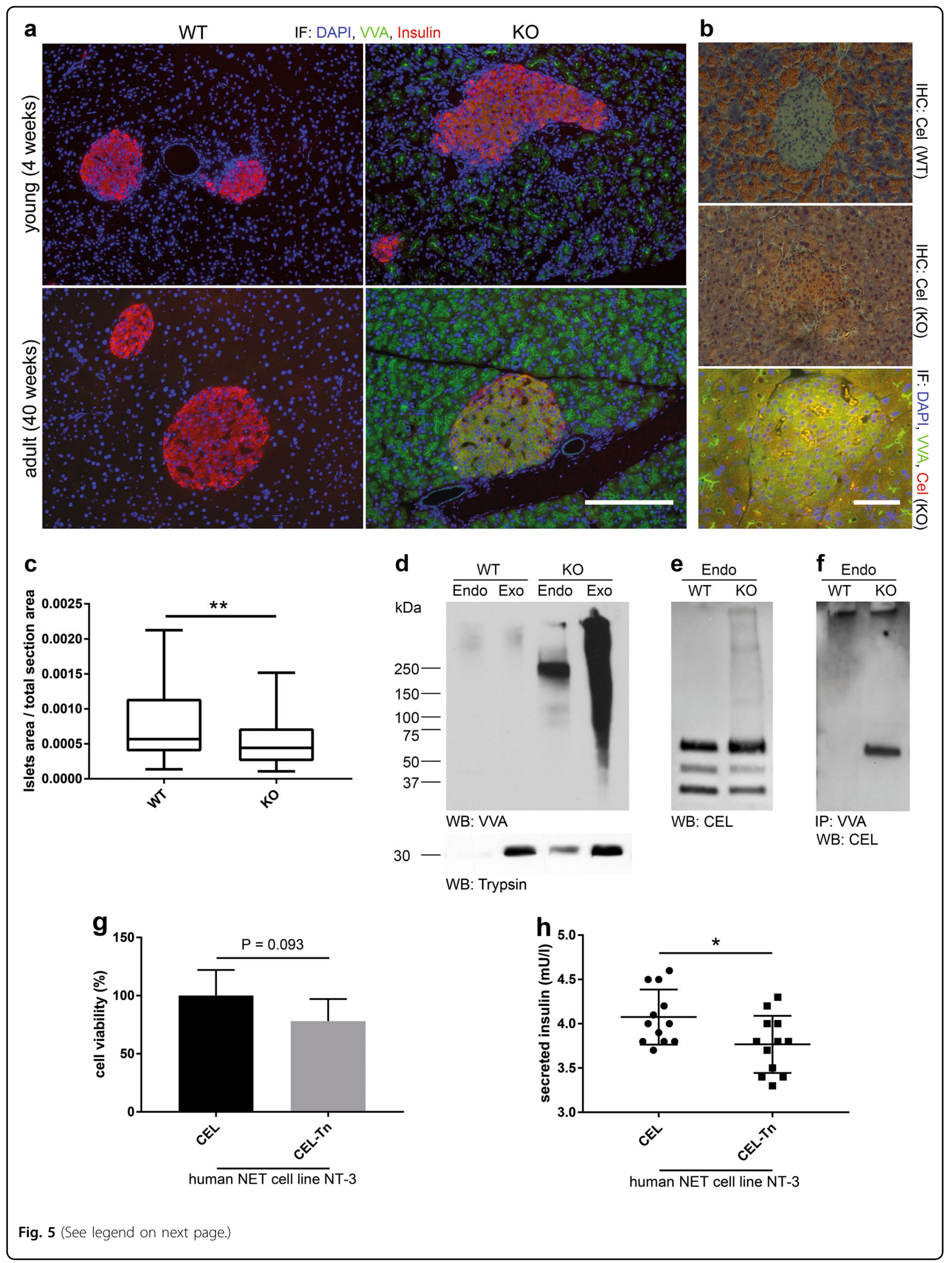


(see figure on previous page)

Fig. 5 Ptf1a-Cre independent accumulation of Tn-modified proteins in pancreatic islets with consecutive beta cell failure. a Assessment of Tn antigen (green) and insulin expression (red) in pancreatic sections from 4 and 40-week-old WT and Cosmc-KO fasting mice. Tn antigen expression is only observed in Cosmc-KO tissue, and Tn antigen positivity is restricted to exocrine glands in young animals, whereas prolonged Tn antigen staining is observed in islets of Langerhans in aged mice. Scale bar equals $200 \mu \mathrm{m}$. b Cel IHC staining of adult WT and KO tissue and IF cO-staining of WA (green) and Cel (red). Scale bar equals $200 \mu \mathrm{m}$. c Quantification of islets of Langerhans of WT $(n=68)$ and KO $(n=64)$ pancreata $(P=0.004)$. d WA Western blot analysis of endo- and exocrine pancreatic tissue derived from WT and Cosmc-KO animals. WA positivity is specific for KO tissue, with most of the Tn-modified proteins found in exocrine tissue. Anti-trypsin was used as an exocrine marker. e Western blot analysis of WT and Cosmc-KO endocrine tissue using anti-Cel antibody. The analysis shows that the localization of Cel is not limited to the exocrine pancreas. $\mathbf{f}$ WA precipitation from endocrine WT and KO tissue lysates with WB detection using anti-Cel antibody. $\mathbf{g}$ Comparison of NT-3 cell viability in the presence of $20 \mu \mathrm{M}$ CEL and CEL-Tn after $48 \mathrm{~h}$ shows no significant difference. $\mathbf{h}$ CEL-Tn shows a significant negative regulation of glucose-stimulated insulin secretion in NT-3 cells. Cells were stimulated with $25 \mathrm{mM}$ glucose for $60 \mathrm{~min}$ in the presence of $20 \mu \mathrm{M}$ recombinant CEL or CEL-Tn

protein response ${ }^{3}$. Interestingly, GRP78 associates with Tsynthase in cancer cell lines deficient in $\mathrm{Cosmc}^{33}$. Generally, O-glycosylation confers resistance to proteolytic degradation, overall protein stability and regulated proteolysis $^{3,34}$. Immature O-glycosylation thus affects regulated zymogen enzyme activation and presumably protein half-life, providing an explanation for the reduced activity of digestive enzymes observed in the present mouse strain.

We utilized the plant lectin VVA to enrich pancreatic Tn antigen glycoproteins that were subsequently analyzed by mass spectrometry. The approach to the identification of O-glycoproteins presented here differs from previous studies (in which genetically modified cell lines were used) by taking biological context and the physiological consequences of altered O-GalNAc glycosylation into account $\mathrm{t}^{3,12}$. The O- glycoproteome has been only partially explored because conserved polypeptide motifs for the prediction of O-glycosylation sites are lacking ${ }^{35}$. Among all the glycan moieties identified as antigens so far, Tn and STn antigens have outstanding potential since both arise in a majority of human cancers ${ }^{36-38}$. Given that over onehalf of all proteins encoded in the human genome are glycosylated $^{39}$, it is not surprising that aberrant glycosylation may have an impact on diverse biological pathways.

$\mathrm{Cel}$, previously described as O-glycosylated ${ }^{15}$, was found consistently among the identified Tn-modified glycoproteins here. Furthermore, our results indicate that the impairment of O-glycosylation on Cel was responsible for the observed MODY8-related phenotype, similar to mutation-induced C-terminal truncation ${ }^{14}$. So far, the pathophysiological mechanisms leading to the disease phenotype are poorly understood, although misfolding and aggregation of mutated $C E L$ have been described to play a pivotal role in the pathogenic process ${ }^{40}$. Ectopically expressed and secreted C-terminal-truncated CEL can be endocytosed by human and rodent cell lines. Internalization of truncated CEL reduces the viability of pancreatic acinar and beta cell models, indicating the induction of apoptosis in pancreatic cells ${ }^{41}$. Our data suggest a pivotal role for the Cel C-terminal region, consisting of a VNTR including PEST sequences enriched in the amino acids proline, glutamate, serine, and threonine and clustered O-glycosylation sites in humans and mice ${ }^{42}$, which are important for normal intracellular protein processing ${ }^{43}$. Notably, the C-terminal domain of Cel harbors neither catalytic ${ }^{44}$ nor regulatory bile salt binding sites ${ }^{45,46}$, and mutation or complete truncation of the latter does not interfere with Cel enzyme activity ${ }^{16}$ Interestingly, the manifestation of MODY8 symptoms in patients is related to the number of VNTRs that include $\mathrm{O}$-glycosylation sites ${ }^{14}$. Another report shows that a recombined allele of CEL and its pseudogene CELP confers susceptibility to chronic pancreatitis. The resulting Cel hybrid protein is characterized by a truncated VNTR region ${ }^{47}$.

In the mouse strain described here, Cel O-glycosylation remained immature, and diabetes onset was observed in mice older than 3 months of age, which is in agreement with previous reports on MODY8 as a progressive disease. The occurrence of pancreatic exocrine dysfunction in MODY8 patients is observed from infancy, and diabetes develops in the fifth decade of life ${ }^{48}$. Despite the fact that endocrine cells, especially insulin-producing beta cells, are not affected by Cosmc deletion in our mouse strain, we observed Tn positivity in adult mice. We further provide evidence for the presence of Cel-Tn in beta cells, causing impairment of insulin secretion and insulin maturation, resulting in reduced serum $\mathrm{C}$-peptide serum in adult Cosmc-KO mice.

In accordance with the reported cluster of symptoms in MODY8 patients ${ }^{14,49}$, characterization of Cosmc-deficient mice revealed symptoms of exocrine pancreatic insufficiency with maldigestion, altered stool formation, impaired zymogen granule release and decreased enzymatic elastase and lipase activity. Moreover, Cosmc-KO mice did not show clinical signs of malnutrition after 8 weeks but had smaller pancreata than control animals. The enzyme activity levels tested were significantly lower, and total cholesterol, triglycerides and HDL decreased in 
blood samples, indicating severely reduced pancreatic acinar function. Fasting serum glucose and $\mathrm{HbA1c}$ were elevated, and fasting serum $\mathrm{C}$-peptide was reduced, consistent with diabetes.

The mouse strain presented here with Cosmc deficiency and loss of core 1 glycans in pancreatic acinar cells showed a causal connection between differential Oglycosylation and exocrine pancreatic dysfunction, as well as diabetes, highlighting the relevance of $\mathrm{O}$ glycosylation to the etiology and pathophysiology of human diseases ${ }^{50-53}$.

\section{Acknowledgements}

This work was supported by grants from the German Research Foundation (DFG) project number 275533756 to GWE and the European Union 7th Framework GlycoBioM Project to CW. BM received funding from United European Gastroenterology. HEK293 SC cells were kindly provided by Prof. H. Clausen (University of Copenhagen, Copenhagen Center for Glycomics). We are grateful to A. Heinecke, P. Schröder, S. Noster and R. Dabels for their excellent technical assistance.

\section{Author details}

'Department of General, Visceral and Thoracic Surgery, University Medical Center Hamburg- Eppendorf (UKE), Hamburg, Germany. ${ }^{2}$ Metabolic Laboratory and Newborn Screening, University Medical Center Hamburg-Eppendorf (UKE), Hamburg, Germany. ${ }^{3}$ Mass Spectrometric Proteomics - Institute for Clinical Chemistry \& Laboratory Medicine, University Medical Center HamburgEppendorf (UKE), Hamburg, Germany. ${ }^{4}$ Center for Diagnostics, University Medical Center Hamburg-Eppendorf (UKE), Hamburg, Germany

\section{Conflict of interest}

The authors declare that they have no conflict of interest.

\section{Publisher's note}

Springer Nature remains neutral with regard to jurisdictional claims in published maps and institutional affiliations.

Supplementary information accompanies this paper at https://doi.org/ 10.1038/s12276-018-0157-3.

Received: 5 August 2017 Revised: 18 June 2018 Accepted: 22 June 2018. Published online: 10 October 2018

\section{References}

1. Agre, P. et al. Training the next generation of biomedical investigators in glycosciences. J. Clin. Invest. 126, 405-408 (2016).

2. Hang, H. C. \& Bertozzi, C. R. The chemistry and biology of mucin-type O-linked glycosylation. Bioorg. Med. Chem. 13, 5021-5034 (2005).

3. Steentoft, C. et al. Precision mapping of the human O-GalNAc glycoproteome through SimpleCell technology. EMBO J. 32, 1478-1488 (2013).

4. Dahr, W., Uhlenbruck, G., Gunson, H. H. \& Van Der Hart, M. Molecular basis of Tn-polyagglutinability. Vox. Sang. 29, 36-50 (1975).

5. Ju, T., Brewer, K, D'Souza, A., Cummings, R. D. \& Canfield, W. M. Cloning and expression of human core 1 beta1,3-galactosyltransferase. J. Biol. Chem. 277, 178-186 (2002).

6. Wang, Y. et al. Cosmc is an essential chaperone for correct protein Oglycosylation. Proc. Natl Acad. Sci. USA 107, 9228-9233 (2010).

7. Sun, Q., Ju, T. \& Cummings, R. D. The transmembrane domain of the molecular chaperone Cosmc directs its localization to the endoplasmic reticulum. J. Biol. Chem. 286, 11529-11542 (2011).

8. Ohtsubo, K. \& Marth, J. D. Glycosylation in cellular mechanisms of health and disease. Cell 126, 855-867 (2006).
9. Obata, J. et al. p48 subunit of mouse PTF1 binds to RBP-Jkappa/CBF-1, the intracellular mediator of Notch signalling, and is expressed in the neural tube of early stage embryos. Genes. Cells 6, 345-360 (2001).

10. Buchakjian, M. R. \& Kornbluth, S. The engine driving the ship: metabolic steering of cell proliferation and death. Nat. Rev. Mol. Cell Biol. 11, 715-727 (2010).

11. Tollefsen, S. E. \& Kornfeld, R. The B4 lectin from Vicia villosa seeds interacts with $\mathrm{N}$ - acetylgalactosamine residues alpha-linked to serine or threonine residues in cell surface glycoproteins. J. Biol. Chem. 258, 5172-5176 (1983).

12. Steentoft, C. et al. Mining the O-glycoproteome using zinc-finger nucleaseglycoengineered SimpleCell lines. Nat. Methods 8, 977-982 (2011).

13. Johansson, B. B. et al. The role of the carboxyl ester lipase (CEL) gene in pancreatic disease. Pancreatology 18, 12-19 (2018).

14. Raeder, H. et al. Mutations in the CEL VNTR cause a syndrome of diabetes and pancreatic exocrine dysfunction. Nat. Genet. 38, 54-62 (2006).

15. Wang, C. S. et al. Isolation and characterization of human milk bile salt-activated lipase C-tail fragment. Biochemistry 34, 10639-10644 (1995).

16. Downs, D., Xu, Y. Y., Tang, J. \& Wang, C. S. Proline-rich domain and glycosylation are not essential for the enzymic activity of bile salt-activated lipase. Kinetic studies of T-BAL, a truncated form of the enzyme, expressed in Escherichia coli. Biochemistry 33, 7979-7985 (1994).

17. Bruneau, N., Nganga, A., Fisher, E. A. \& Lombardo, D. O-Glycosylation of Cterminal tandem-repeated sequences regulates the secretion of rat pancreatic bile salt-dependent lipase. J. Biol. Chem. 272, 27353-27361 (1997).

18. Neuman, J. C., Truchan, N. A., Joseph, J. W. \& Kimple, M. E. A method for mouse pancreatic islet isolation and intracellular CAMP determination. J. Vis. Exp. 88, e50374 (2014).

19. Gout J., et al. Isolation and culture of mouse primary pancreatic acinar cells. J. Vis. Exp. 78 (2013).

20. Rindler M. J. Isolation of zymogen granules from rat pancreas. In: Bonifacino J. S., Basso M., Harford J. B., Lippincott-Schwartz J., Yamada K. M. (eds). Current Protocols in Cell Biology. John Wiley \& Sons, Inc.: New York, 2006; Vol. 1, pp 3.18.1-3.18.15.

21. Hofmann, B. T. et al. COSMC knockdown mediated aberrant O-glycosylation promotes oncogenic properties in pancreatic cancer. Mol. Cancer 14, 109 (2015).

22. Lutz, D., Wolters-Eisfeld, G., Schachner, M. \& Kleene, R. Cathepsin E generates a sumoylated intracellular fragment of the cell adhesion molecule L1 to promote neuronal and Schwann cell migration as well as myelination. J. Neurochem. 128, 713-724 (2014)

23. Wolters-Eisfeld, G. \& Schumacher, U. Lectin histochemistry for metastasizing and non-metastasizing cancer cells. Methods Mol. Biol. 1560, 121-132 (2017).

24. Benten, D. et al. Establishment of the first well-differentiated human pancreatic neuroendocrine tumor model. Mol. Cancer Res. 16, 496-507 (2018).

25. Ju, T. et al. A novel fluorescent assay for T-synthase activity. Glycobiology 21, 352-362 (2011)

26. Vembar, S. S. \& Brodsky, J. L. One step at a time: endoplasmic reticulumassociated degradation. Nat. Rev. Mol. Cell Biol. 9, 944-957 (2008).

27. Song, K. et al. Loss of mucin-type O-glycans impairs the integrity of the glomerular filtration barrier in the mouse kidney. J. Biol. Chem. 292 16491-16497 (2017).

28. Kudelka, M. R. et al. Cosmc is an X-linked inflammatory bowel disease risk gene that spatially regulates gut microbiota and contributes to sex-specific risk. Proc. Natl Acad. Sci. USA 113, 14787-14792 (2016).

29. De Lisle, R. C. Role of sulfated O-linked glycoproteins in zymogen granule formation. J. Cell. Sci. 115(Pt 14), 2941-2952 (2002).

30. De Lisle, R. C. \& Ziemer, D. Processing of pro-Muclin and divergent trafficking of its products to zymogen granules and the apical plasma membrane of pancreatic acinar cells. Eur. J. Cell Biol. 79, 892-904 (2000).

31. De Lisle, R. C., Xu, W., Roe, B. A. \& Ziemer, D. Effects of Muclin (Dmbt1) deficiency on the gastrointestinal system. Am. J. Physiol. Gastrointest. 294, G717-G727 (2008).

32. Lee, A. S. The ER chaperone and signaling regulator GRP78/BiP as a monitor of endoplasmic reticulum stress. Methods 35, 373-381 (2005).

33. Ju, T., Aryal, R. P., Stowell, C. J. \& Cummings, R. D. Regulation of protein Oglycosylation by the endoplasmic reticulum-localized molecular chaperone Cosmc. J. Cell. Biol. 182, 531-542 (2008). 
34. Rogers, S., Wells, R. \& Rechsteiner, M. Amino acid sequences common to rapidly degraded proteins: the PEST hypothesis. Science 234, 364-368 (1986).

35. Julenius, K., Molgaard, A., Gupta, R. \& Brunak, S. Prediction, conservation analysis, and structural characterization of mammalian mucin-type O-glycosylation sites. Glycobiology 15, 153-164 (2005).

36. Kim, G. E. et al. Aberrant expression of MUC5AC and MUC6 gastric mucins and sialyl Tn antigen in intraepithelial neoplasms of the pancreas. Gastroenterology 123, 1052-1060 (2002).

37. Springer, G. F. T. and Tn, general carcinoma autoantigens. Science 224, 1198-1206 (1984).

38. Terada, T. \& Nakanuma, Y. Expression of mucin carbohydrate antigens $(T, T n$ and sialyl $T n$ ) and MUC-1 gene product in intraductal papillarymucinous neoplasm of the pancreas. Am. J. Clin. Pathol. 105, 613-620 (1996).

39. Zhang, $H$. et al. Simultaneous characterization of glyco- and phosphoproteomes of mouse brain membrane proteome with electrostatic repulsion hydrophilic interaction chromatography. Mol. Cell. Proteom. 9, 635-647 (2010).

40. Johansson, B. B. et al. Diabetes and pancreatic exocrine dysfunction due to mutations in the carboxyl ester lipase gene-maturity onset diabetes of the young (CEL-MODY): a protein misfolding disease. J. Biol. Chem. 286, 34593-34605 (2011)

41. Torsvik, J. et al. Endocytosis of secreted carboxyl ester lipase in a syndrome of diabetes and pancreatic exocrine dysfunction. J. Biol. Chem. 289, 29097-29111 (2014).

42. Higuchi, S., Nakamura, Y. \& Saito, S. Characterization of a VNTR polymorphism in the coding region of the CEL gene. J. Hum. Genet. 47, 213-215 (2002)
43. Lombardo, D. Bile salt-dependent lipase: its pathophysiological implications Biochim. Biophys. Acta 1533, 1-28 (2001).

44. DiPersio, L. P., Fontaine, R. N. \& Hui, D. Y. Identification of the active site serine in pancreatic cholesterol esterase by chemical modification and site-specific mutagenesis. J. Biol. Chem. 265, 16801-16806 (1990).

45. Aubert, E., Sbarra, V., Le Petit-Thevenin, J., Valette, A. \& Lombardo, D. Sitedirected mutagenesis of the basic N-terminal cluster of pancreatic bile saltdependent lipase. Functional significance. J. Biol. Chem. 277, 34987-34996 (2002).

46. Aubert-Jousset, E., Sbarra, V. \& Lombardo, D. Site-directed mutagenesis of the distal basic cluster of pancreatic bile salt-dependent lipase. J. Biol. Chem. 279 39697-39704 (2004)

47. Fjeld, K. et al. A recombined allele of the lipase gene CEL and its pseudogene CELP confers susceptibility to chronic pancreatitis. Nat. Genet. 47, 518-522 (2015).

48. Raeder, H. et al. Carboxyl-ester lipase maturity- onset diabetes of the young is associated with development of pancreatic cysts and upregulated MAPK signaling in secretin-stimulated duodenal fluid. Diabetes 63, 259-269 (2014).

49. Tjora, E. et al. Severe pancreatic dysfunction but compensated nutritional status in monogenic pancreatic disease caused by carboxyl-ester lipase mutations. Pancreas 42, 1078-1084 (2013).

50. Fu, J. et al. Loss of intestinal core 1-derived O- glycans causes spontaneous colitis in mice. J. Clin. Invest. 121, 1657-1666 (2011).

51. Ju, T. \& Cummings, R. D. Protein glycosylation: chaperone mutation in $\mathrm{Tn}$ syndrome. Nature 437, 1252 (2005).

52. Wang, $Y$. et al. Platelet biogenesis and functions require correct protein $\mathrm{O}-$ glycosylation. Proc. Natl Acad. Sci. USA 109, 16143-16148 (2012).

53. Bjorkqvist, J. et al. Defective glycosylation of coagulation factor XII underlies hereditary angioedema type III. J. Clin. Invest. 125, 3132-3146 (2015). 